\title{
The neutrophil-to-lymphocyte and monocyte-to-lymphocyte ratios are independently associated with neurological disability and brain atrophy in multiple sclerosis
}

\author{
Christopher C. Hemond ${ }^{1,2,3^{*}}$ (D), Bonnie I. Glanz ${ }^{2,3}$, Rohit Bakshi ${ }^{2,3,4}$, Tanuja Chitnis ${ }^{2,3}$ and Brian C. Healy ${ }^{2,3}$
}

\begin{abstract}
Background: Serum hematological indices such as the neutrophil-lymphocyte ratio (NLR) or monocyte-lymphocyte ratio (MLR) have been used as biomarkers of pathogenic inflammation and prognostication in multiple areas of medicine; recent evidence shows correlation with psychological parameters as well.

Objectives/Aims: To characterize clinical, neuroimaging, and psycho-neuro-immunological associations with NLR and MLR in persons with multiple sclerosis (MS).

Methods: We identified a large cohort of clinically well-defined patients from our longitudinal database that included MS-related outcomes, disease-modifying therapy, patient-reported outcome (PRO) measures, and quantified cerebral MRI at 1.5 T. We queried hospital records for complete blood counts within 2 months of each clinic visit and excluded those obtained during clinical relapses. Four hundred eighty-three patients, with a mean of 3 longitudinal observations each, were identified who met these criteria. Initial analyses assessed the association between NLR and MLR as the outcomes, and psychological and demographic predictors in univariable and multivariable models controlling for age, gender and treatment. The second set of analyses assessed the association between clinical and MRI outcomes including whole brain atrophy and T2-hyperintense lesion volume, with NLR and MLR as predictors in univariable and multivariable models. All analyses used a mixed effects linear or logistic regression model with repeated measures.
\end{abstract}

Results: Unadjusted analyses demonstrated significant associations between higher (log-transformed) NLR (but not MLR) and PRO measures including increasing depression $(p=0.01)$, fatigue $(p<0.01)$, and decreased physical quality of life $(p<0.01)$. Higher NLR and MLR strongly predicted increased MS-related disability as assessed by the Expanded Disability Status Scale, independent of all demographic, clinical, treatment-related, and psychosocial variables $(p<0.001)$. Lastly, higher NLR and MLR significantly discriminated progressive from relapsing status ( $p \leq 0.01$ for both), and higher MLR correlated with increased whole-brain atrophy $(p<0.05)$ but not T2 hyperintense lesion volume $(p>0.05)$ even after controlling for all clinical and demographic covariates. Sensitivity analyses using a subset of untreated patients $(N=146)$ corroborated these results.

(Continued on next page)

\footnotetext{
* Correspondence: christopher.hemond@umassmed.edu

'Department of Neurology, University of Massachusetts Medical Center, 55 Lake Ave North, Worcester, MA 01655, USA

${ }^{2}$ Department of Neurology, Harvard Medical School, Boston, MA, USA

Full list of author information is available at the end of the article
}

(c) The Author(s). 2019 Open Access This article is distributed under the terms of the Creative Commons Attribution 4.0 International License (http://creativecommons.org/licenses/by/4.0/), which permits unrestricted use, distribution, and reproduction in any medium, provided you give appropriate credit to the original author(s) and the source, provide a link to the Creative Commons license, and indicate if changes were made. The Creative Commons Public Domain Dedication waiver (http://creativecommons.org/publicdomain/zero/1.0/) applies to the data made available in this article, unless otherwise stated. 
(Continued from previous page)

Conclusions: Elevated NLR and MLR may represent hematopoetic bias toward increased production and proinflammatory priming of the myeloid innate immune system (numerator) in conjunction with dysregulated adaptive immune processes (denominator), and consequently reflect a complementary and independent marker for severity of MS-related neurological disability and MRI outcomes.

Keywords: Multiple sclerosis, Neutrophil-to-Lympocyte ratio, Monocyte-to-Lympocyte ratio, Brain atrophy, MRI, Patientreported outcomes,

\section{Background} Introduction

Multiple sclerosis (MS) is a progressive neurodegenerative condition of presumed autoimmune etiology typically characterized by recurrent episodes of inflammatory demyelination and accelerated tissue destruction of the central nervous system. The etiology of the disease is thought to principally involve the peripheral activation of Th1 lymphocytes which traffic to the central nervous system and react with myelin autoantigens. Following activation, these T-cells recruit a diverse set of myeloid cells including monocytes/macrophages to promote and execute an inflammatory reaction, frequently causing axonal transection and irreversible focal central nervous system (CNS) injury [1]. In addition to monocytes, neutrophils have also recently been implicated to play a role in augmentation of the inflammatory response in MS [2].

The neutrophil-lymphocyte ratio (NLR) and monocyte-lymphocyte ratio (MLR) are inexpensive and readily available components of the standard complete blood count $(\mathrm{CBC})$; they are increasingly recognized as clinically-relevant biomarkers of pathological inflammation across multiple areas of medicine including oncology [3, 4], cardiovascular disease [5], and autoimmune conditions [6]. In neurology, NLR improves outcome prediction in stroke $[7,8]$, and is elevated compared to healthy controls in degenerative conditions like Alzheimer's $[9,10]$ and Parkinson's [11] disease.

The NLR and MLR have also been linked to psychological parameters. The NLR has a well-established short-term response to both endogenous and exogenous corticosteroids [12] and links to perceived stress in humans [13]. A recent meta-analysis concluded that there is an elevated NLR in several mood disorders including depression [14]. In a population of patients with stable coronary artery disease, Serfozo and colleagues showed a strong independent correlation (after controlling for age, body mass index and sex) between the MLR and depression symptom severity score, as well as other inflammatory markers (IL-6, C-Reactive Protein), and neuroendocrine-sympathetic activity as measured by chromogranin A [15]. It has been proposed that psychosocial stress and related chronic psychiatric diseases (depression, anxiety) may bias the hemopoetic differentiation process toward both (1) increased production of monocytes and granulocytes and (2) enhance myeloid pro-inflammatory 'priming' and trafficking, both via bidirectional interactions between CNS sympathic/neuroendocrine systems and lymphoid tissue [16-18]. Irrespective of psychosocial factors however, both neutrophils and monocytes have been observed to be 'primed' for pro-inflammatory activity in persons with multiple sclerosis, thought to be related to the chronic systemic inflammatory environment of this disease $[2,19]$.

There is limited prior literature regarding the NLR in MS; Demirci and colleagues were the first to explore this area and found that an elevated NLR could discriminate MS from healthy controls, was related to neurological disability scores, and was further increased in flares compared to remission [20]. Several subsequent studies have confirmed similar findings [13, 20, 21], although one study could not replicate associations with disability scores [22]. None of these studies carefully examined the role of disease-modifying therapies however; nor did they look at MRI-related disease outcomes such as cerebral atrophy or quantified T2-hyperintense lesion volume. The role of MLR has not been studied in MS to our knowledge. This work has been previously published in abstract format [23].

\section{Objectives/aims}

Here we hypothesize that the NLR and MLR are psycho-neuro-immunological markers; modulated in part by factors such as stress, depression, and social isolation/ support. Furthermore, we hypothesize that the NLR and MLR play a role in MS-related disability by contributing to (or reflecting) a pro-inflammatory state. To this end, our first aim was to establish whether a relationship exists between the NLR/MLR and demographic variables including age and sex, as well as MS-related clinical phenotypes, disease duration, and disease-modifying therapies. Second, we sought to better understand associations between the NLR/MLR and patient-reported psychological outcomes including mental health, social support, depression, and quality of life. Third, we aimed to explore associations with objective markers of neuroinflammation and degeneration as reflected by neurological disability and MRI neuroimaging parameters relevant in MS, including 
whole brain atrophy and total burden of cerebral T2-hyperintense lesions.

\section{Methods Subjects}

This is a retrospective analysis of prospectively-collected data in a cohort of multiple sclerosis subjects who participated in the Comprehensive Longitudinal Investigation of Multiple Sclerosis at the Brigham and Women's Hospital, Partners MS Center (CLIMB). CLIMB is an ongoing, prospective observational cohort study, started in 2000 [24]. General inclusion criteria to participate include: age $\geq 18$ years and a diagnosis of relapsing-spectrum or progressive MS syndrome based on revised McDonald Criteria [25]. MS participants are examined at a minimum of every 12 months by a neurologist with subspecialty MS training; demographic and clinical data are regularly collected as part of this study. Additionally, a subset of patients consented to complete patient-reported outcome measures (PRO) as detailed below. Additional inclusion criteria for this specific study were: age $<65$, and a $C B C$ with differential within 60 days of their patient-reported outcome assessment. Exclusion criteria included: CBC-d obtained on the same day or the following 1 month after reported clinical flare, or multiple CBCs within 4 days of each other which were thought to have a high probability of being obtained during times of infection or inpatient hospitalization. In total, 483 patients were included in the analysis, with a mean of 3.0 observations each (range: 1-10) for a total of 1433 observations. Subject demographics, clinical characteristics, patient self-reported outcome measures, and neuroimaging data are summarized in Table 1.

\section{Consent to participate}

This study was reviewed and approved by the Partners Human Research Committee ethics board at the Brigham and Women's Hospital (IRB Protocol \# 1999-P-010435). All research subjects provided informed written consent.

\section{Patient-reported measures}

Four patient-reported psychological questionnaires are administered to a subset of participants in the CLIMB study as follows. The 20-item Center for Epidemiology Depression scale (CES-D) [26] is a validated scale that focuses on the cognitive and affective rather than the somatic components of depression. Scores range from 20 to 80 and a cutoff of $\geq 36$ is considered to be in the depressed range. The 21-item Modified Fatigue Inventory Scale (MFIS) [27] assesses physical, cognitive, and psychosocial fatigue with a score range from 0 to 84; a cutoff of $\geq 38$ represents clinically meaningful fatigue. The SF-36 [28] is an inventory of mental, physical, and social measures of life satisfaction and fitness that assesses eight health concepts and is summarized in two composite scores of physical and mental quality of life. The 18-item Medical Outcomes Modified Study Social Support Survey (MSSS) [29] is an assessment of patient's perceived social support with four subscales and raw scores ranging from 18 to 90 . PROs were administered annually at study inception (2000) until 2011 when they began being collected biennially. The exception is the MFIS, which was added on 6/23/2006.

\section{Complete blood counts}

Automated complete blood counts with differential were obtained via an electronic query of hospital records. Personal communication with hematology, as well as reference test numbers, confirmed the use of two automated hematological analysis machines during the data collection period: Siemens ADVIA 2120 was used prior to 10/ 29/2011 and a SYSMEX XE 5000 was used after 10/29/ 2011. The vast majority of the data were from the ADVIA 2120, so measurements from the SYSMEX XE were excluded from the study to reduce variability (see Discussion).

\section{Neuroimaging}

Brain MRI scans were acquired at $1.5 \mathrm{~T}$ from several Signa GE scanners with similar dual-echo spin-echo acquisition parameters: TR $=2800-3000 \mathrm{msec}, \mathrm{TE} 1 / \mathrm{TE} 2=30 / 80$ msec, slice thickness $=3 \mathrm{~mm}$ (gapless), pixel size $=0.93 \times$ $0.93 \mathrm{~mm}$. Brain parenchymal fraction (BPF) and cerebral T2-hyperintense lesion volumes (T2LV) were obtained from automated template-driven segmentation [30] with additional expert manual correction as required. Brain parenchymal fraction was defined as the summation of cerebral gray and white matter divided by volume of the intracranial cavity to yield a self-normalized fraction. T2LV was quantified a summated single volume measurement.

\section{Statistical analysis}

Statistical analysis of the demographic, clinical, and laboratory variables was performed using the $\mathrm{R}$ (http:// www.R-project.org) or Stata v14.0 (www.stata.com) software. Normality of variables was assessed by a combination of skew/kurtosis metrics, visual inspection of the histogram distribution and the Shapiro-Wilk normality test. NLR/MLR did not meet normality criteria and was therefore natural $\log$ (base $e$ ) transformed. Most other variables were skewed so medians and interquartile ranges are reported as opposed to means and standard deviations. In our first set of analyses, we assessed which demographic, treatment, and psychosocial variables were associated with the concurrent natural log-transformed NLR or MLR. For this analysis, we accounted for 
Table 1 Clinical and demographic characteristics, neuroimaging, and patient-reported outcomes

\begin{tabular}{|c|c|}
\hline Variable & Median (IQR) or N (\%) \\
\hline $\mathrm{N}$ & 483 \\
\hline Age (years) & $44.5(36,51.9)$ \\
\hline Disease Duration (years) & $8.8(5.3,16)$ \\
\hline Neutrophil-lymphocyte ratio & $2.1(1.54,2.9)$ \\
\hline Monocyte-lymphocyte ratio & $0.2(0.15,0.28)$ \\
\hline Expanded Disability Status Scale & $2(1,3)$ \\
\hline \multicolumn{2}{|l|}{ Gender } \\
\hline - Female & $347(71.8 \%)$ \\
\hline - Male & $136(28.2 \%)$ \\
\hline \multicolumn{2}{|l|}{ Smoking History ${ }^{a}$} \\
\hline$\cdot \mathrm{No}$ & $233(54.2 \%)$ \\
\hline - Yes & $197(45.8 \%)$ \\
\hline Body mass index (BMI) & $26.2(22.8,31.1)$ \\
\hline \multicolumn{2}{|l|}{ Multiple sclerosis disease category } \\
\hline - Clinically isolated syndrome & $15(3.1 \%)$ \\
\hline - Relapsing-remitting & $370(76.6 \%)$ \\
\hline - Secondary progressive & $65(13.5 \%)$ \\
\hline - Primary progressive & $33(6.8 \%)$ \\
\hline \multicolumn{2}{|l|}{ Disease modifying therapy } \\
\hline - Untreated & $110(22.8 \%)$ \\
\hline • Interferon- $\beta$ & $216(44.7 \%)$ \\
\hline - Cyclophosphamide & $14(2.9 \%)$ \\
\hline • Fingolimod & $8(1.7 \%)$ \\
\hline - Glatiramer Acetate & $51(10.6 \%)$ \\
\hline - Mycophenolate Mofetil & $33(6.8 \%)$ \\
\hline - Natalizumab & $37(7.7 \%)$ \\
\hline - Other & $14(2.9 \%)$ \\
\hline Neuroimaging and patient-reported outcome measures & Median (IQR); N \\
\hline Brain parenchymal fraction & $0.85(0.81,0.88) ; 334$ \\
\hline Cerebral T2-hyperintense lesion volume (mL) & $1.57(1.27,2.03) ; 334$ \\
\hline Center for Epidemiological Studies Depression Scale & $29(24,36) ; 479$ \\
\hline Modified Fatigue Inventory Scale & $29(14,41) ; 425$ \\
\hline Modified Social Support Scale & $85.4(71.1,98.4) ; 477$ \\
\hline SF-36 Physical composite & $46.6(37.9,54.3) ; 471$ \\
\hline SF-36 Mental composite & $50.9(42.5,56.4) ; 471$ \\
\hline
\end{tabular}

Legend: "Other" DMT includes combinations of or more of the following: methotrexate, azathioprine, pulsed glucocorticoids, anti-IL2 agents, rituximab, natalizumab, interferons, glatiramer acetate, cyclophosphamide. Cyclophosphamide, methotrexate and mycophenolate mofetil were occasionally combined with an interferon or glatiramer acetate; otherwise medications are listed as monotherapy. Median and interquartile ranges use data from patient's most recent available clinical visit. ${ }^{a}$ Smoking history $(N=430)$, BMI $(N=402)$

repeated observations on subjects using a linear mixed effects model with a subject-specific random intercept. Each potential predictor was included in the model separately in the univariable analysis. Further, a multivariable model including demographic factors, smoking history and specific disease-modifying therapy (DMT) use together was also fit to estimate the independent association of each feature accounting for these covariates. In addition to the analysis including all measurements per subject, a separate univariate analysis including only the most recent time point was performed as well, and included as Additional file 1 . In the second set of analyses, we assessed whether NLR or MLR was associated with clinical and radiologic 
measures of disease severity using a linear mixed effects model with a subject specific random intercept. In this model, the EDSS, BPF, cubic-transformed T2 lesion volume, and clinical disease category were the outcome measures. We assessed the univariable association including only NLR or MLR, and we also fit 2 different multivariable models. Model 1 included demographic and treatment predictors, whereas model 2 included demographic, treatment and psychosocial predictors to assess the independent contribution of NLR and MLR on the outcomes. Lastly, we assessed whether baseline NLR or MLR could predict subsequent (longitudinal) worsening of a patient's MS-related disability using EDSS as the outcome variable in a linear mixed effects model with repeated measures. In this model the predictor variables included time elapsed (days) since baseline measurement, the natural log-transformed MLR or NLR, and the interaction between the two maintained as continuous variables.

\section{Results}

Clinical, demographic, and self-reported outcomes associated with the NLR and MLR

Univariable and multivariable associations between demographic and psychosocial measures with NLR are provided in Table 2, and MLR in Table 3. In both univariable and multivariable comparisons controlling for age, gender, smoking history, and DMT, NLR was significantly higher for subjects taking cyclophosphamide and fingolimod, and significantly lower for subjects taking interferons and natalizumab. Increased NLR was also significantly associated with higher depression (CES-D) scores, higher fatigue scores (MFIS), and lower physical quality of life (SF-36), but not social support as assessed by the MSSS, or BMI (both $p>0.05$ ). Uni- and multi-variable analyses with MLR as the outcome showed similar significant associations with the same DMTs as NLR except directionality reversal for interferons; additionally, higher MLR significantly correlates with older age, longer disease duration (univariable

Table 2 Clinical, demographic, and patient self-reported associations with the (natural log-transformed) Neutrophil-Lymphocyte Ratio

\begin{tabular}{|c|c|c|c|c|c|c|}
\hline & \multicolumn{3}{|c|}{ Univariable } & \multicolumn{3}{|c|}{ Multivariable } \\
\hline & $\beta$ & $95 \% \mathrm{Cl}$ & $p$ & $\bar{\beta}$ & $95 \% \mathrm{Cl}$ & $p$ \\
\hline Age (yrs) & 0.023 & $-0.011,0.056$ & 0.188 & 0.021 & $-0.010,0.053$ & 0.189 \\
\hline Disease Duration (yrs) & 0.009 & $-0.023,0.040$ & 0.591 & -0.013 & $-0.048,0.022$ & 0.477 \\
\hline \multicolumn{7}{|l|}{ Gender } \\
\hline - Female & - & - & - & - & - & - \\
\hline - Male & -0.003 & $-0.083,0.077$ & 0.942 & -0.012 & $-0.086,0.062$ & 0.750 \\
\hline \multicolumn{7}{|l|}{ Smoking History } \\
\hline - No & - & - & - & - & - & - \\
\hline - Yes & 0.001 & $-0.076,0.078$ & 0.979 & 0.018 & $-0.054,0.090$ & 0.621 \\
\hline $\mathrm{BMI}$ & -0.008 & $-0.045,0.029$ & 0.666 & 0.002 & $-0.033,0.037$ & 0.910 \\
\hline \multicolumn{7}{|l|}{ DMT } \\
\hline - Untreated & - & - & - & - & - & - \\
\hline - Interferon- $\beta$ & -0.166 & $-0.236,-0.096$ & $<0.001$ & -0.163 & $-0.233,-0.093$ & $<0.001$ \\
\hline - Cyclophosphamide & 0.268 & $0.147,0.389$ & $<0.001$ & 0.274 & $0.152,0.395$ & $<0.001$ \\
\hline - Fingolimod & 1.044 & $0.767,1.320$ & $<0.001$ & 1.047 & $0.770,1.323$ & $<0.001$ \\
\hline - Glatiramer Acetate & -0.082 & $-0.187,0.024$ & 0.131 & -0.079 & $-0.185,0.027$ & 0.142 \\
\hline - Mycophenolate & -0.072 & $-0.181,0.037$ & 0.197 & -0.075 & $-0.184,0.035$ & 0.181 \\
\hline • Natalizumab & -0.529 & $-0.647,-0.411$ & $<0.001$ & -0.526 & $-0.644,-0.408$ & $<0.001$ \\
\hline - Other & -0.009 & $-0.141,0.124$ & 0.900 & -0.012 & $-0.144,0.121$ & 0.862 \\
\hline CES-D & 0.034 & $0.008,0.061$ & 0.012 & 0.030 & $0.004,0.055$ & 0.022 \\
\hline MFIS & 0.045 & $0.012,0.078$ & 0.008 & 0.035 & $0.004,0.066$ & 0.029 \\
\hline MSSS & -0.019 & $-0.047,0.010$ & 0.203 & -0.021 & $-0.048,0.006$ & 0.121 \\
\hline SF-36 Physical QOL & -0.055 & $-0.086,-0.024$ & $<0.001$ & -0.037 & $-0.067,-0.007$ & 0.015 \\
\hline SF-36 Mental QOL & -0.024 & $-0.050,0.003$ & 0.086 & -0.024 & $-0.049,0.002$ & 0.066 \\
\hline
\end{tabular}

Legend: CES-D Center for epidemiology scale for depression, MFIS Modified fatigue impact scale, MSSS Modified social support survey, QOL Quality of life, BMI Body mass index, DMT Disease modifying therapy. Mixed linear regression models use data from all patient visits (repeated measures), with multivariable model including the following covariates: age, gender, smoking history, and DMT use. Continuous predictor variables are Z-transformed such that one unit increase represents one standard deviation change. Bolded values represent $p<0.05$ 
Table 3 Clinical, demographic, and patient self-reported associations with the (natural log-transformed) Monocyte-lymphocyte Ratio

\begin{tabular}{|c|c|c|c|c|c|c|}
\hline & \multicolumn{3}{|c|}{ Univariable } & \multicolumn{3}{|c|}{ Multivariable } \\
\hline & $\bar{\beta}$ & $95 \% \mathrm{Cl}$ & $p$ & $\bar{\beta}$ & $95 \% \mathrm{Cl}$ & $p$ \\
\hline Age (yrs) & 0.051 & $0.019,0.082$ & 0.002 & 0.057 & $0.028,0.086$ & $<0.001$ \\
\hline Disease Duration (yrs) & 0.043 & $0.013,0.072$ & 0.004 & 0.033 & $0.000,0.065$ & 0.049 \\
\hline \multicolumn{7}{|l|}{ Gender } \\
\hline - Female & - & - & - & - & - & - \\
\hline - Male & 0.163 & $0.090,0.237$ & $<0.001$ & 0.160 & $0.092,0.229$ & $<0.001$ \\
\hline \multicolumn{7}{|l|}{ Smoking History } \\
\hline • No & - & - & - & - & - & - \\
\hline - Yes & -0.002 & $-0.074,0.070$ & 0.958 & 0.003 & $-0.063,0.069$ & 0.937 \\
\hline BMI & -0.031 & $-0.066,0.003$ & 0.074 & -0.024 & $-0.056,0.007$ & 0.132 \\
\hline \multicolumn{7}{|l|}{ DMT } \\
\hline - Untreated & - & - & - & - & - & - \\
\hline - Interferon- $\beta$ & 0.068 & $0.004,0.132$ & 0.038 & 0.077 & $0.013,0.140$ & 0.018 \\
\hline - Cyclophosphamide & 0.296 & $0.187,0.406$ & $<0.001$ & 0.310 & $0.201,0.418$ & $<0.001$ \\
\hline - Fingolimod & 1.219 & $0.970,1.469$ & $<0.001$ & 1.207 & $0.959,1.454$ & $<0.001$ \\
\hline - Glatiramer Acetate & -0.045 & $-0.142,0.052$ & 0.360 & -0.047 & $-0.142,0.049$ & 0.339 \\
\hline - Mycophenolate & 0.062 & $-0.037,0.162$ & 0.219 & 0.063 & $-0.035,0.162$ & 0.206 \\
\hline - Natalizumab & -0.378 & $-0.486,-0.270$ & $<0.001$ & -0.374 & $-0.48,-0.267$ & $<0.001$ \\
\hline - Other & 0.057 & $-0.063,0.178$ & 0.350 & 0.048 & $-0.071,0.168$ & 0.426 \\
\hline CES-D & 0.010 & $-0.015,0.034$ & 0.437 & 0.007 & $-0.016,0.030$ & 0.534 \\
\hline MFIS & 0.008 & $-0.022,0.038$ & 0.604 & 0.005 & $-0.022,0.033$ & 0.704 \\
\hline MSSS & 0.007 & $-0.019,0.033$ & 0.604 & 0.006 & $-0.018,0.031$ & 0.611 \\
\hline SF-36 Physical QOL & -0.017 & $-0.045,0.012$ & 0.254 & -0.011 & $-0.038,0.016$ & 0.441 \\
\hline SF-36 Mental QOL & -0.005 & $-0.030,0.019$ & 0.676 & -0.005 & $-0.027,0.018$ & 0.678 \\
\hline
\end{tabular}

Legend: CES-D Center for epidemiology scale for depression, MFIS Modified fatigue impact scale, MSSS Modified social support survey, QOL Quality of life, $B M I$ Body mass index, DMT Disease modifying therapy. Mixed linear regression models use data from all patient visits (repeated measures), with multivariable model including the following covariates: age, gender, smoking history, and DMT use. Continuous predictor variables are Z-transformed such that one unit increase represents one standard deviation change. Bolded values represent $p<0.05$

$p<0.01$; multivariable $p=0.052$ ) and male gender, but not with any patient-reported outcome measures.

\section{NLR/MLR are associated with MS-related disability and neuroimaging outcomes}

We then examined disease category (progressive vs. relapsing), physical disability status (EDSS score), and neuroimaging outcome measures (BPF and $\mathrm{T} 2 \mathrm{LV}$ ) with results summarized in Tables 4 and 5 . In all models, higher NLR and MLR were both robustly associated with increased EDSS scores; effect sizes were slightly larger using MLR $(\beta \sim 0.26)$ compared to NLR $(\beta \sim 0.21)$. Higher NLR and MLR both significantly discriminated progressive from relapsing forms of MS in univariable and basic multivariable analyses, although only NLR significantly added to the complex multivariable analysis

Table 4 Natural log-transformed neutrophil-lymphocyte ratio as a predictor of objective MS disease outcomes

\begin{tabular}{|c|c|c|c|c|c|c|c|c|c|}
\hline \multirow[t]{2}{*}{ Outcome } & \multicolumn{3}{|c|}{ Univariable } & \multicolumn{3}{|c|}{ Multivariable Model 1} & \multicolumn{3}{|c|}{ Multivariable Model 2} \\
\hline & $\bar{\beta}$ & $95 \% \mathrm{Cl}$ & $p$ & $\beta$ & $95 \% \mathrm{Cl}$ & $p$ & $\beta$ & $95 \% \mathrm{Cl}$ & $p$ \\
\hline EDSS & 0.208 & $0.078,0.338$ & 0.002 & 0.238 & $0.106,0.369$ & $<0.001$ & 0.217 & $0.082,0.351$ & 0.002 \\
\hline RRMS $\vee$ PMS & 0.219 & $0.093,0.344$ & 0.001 & 0.599 & $0.350,0.849$ & $<0.001$ & 0.452 & $0.157,0.747$ & 0.003 \\
\hline BPF & -0.001 & $-0.004,0.002$ & 0.610 & -0.001 & $-0.004,0.001$ & 0.243 & -0.002 & $-0.004,0.001$ & 0.156 \\
\hline T2LV & -0.001 & $-0.032,0.031$ & 0.970 & 0.002 & $-0.031,0.035$ & 0.912 & 0.006 & $-0.029,0.041$ & 0.753 \\
\hline
\end{tabular}

Legend: EDSS Expanded disability status scale, RRMS Relapsing-remitting multiple sclerosis, PMS Progressive multiple sclerosis, BPF Brain parenchymal fraction, T2LV Cerebral T2-hyperintense lesion volume. All statistical models are linear mixed effects regressions. Multivariable model \#1 includes the following covariates: age, disease duration, gender, and specific DMT use. Model \#2 includes the covariates: age, disease duration, gender, smoking history, specific DMT use, CES-D score, MSSS score, SF-36 physical and mental composite scores 
Table 5 Natural log-transformed monocyte-lymphocyte ratio as a predictor of objective MS disease outcomes

\begin{tabular}{|c|c|c|c|c|c|c|c|c|c|}
\hline \multirow[t]{2}{*}{ Outcome } & \multicolumn{3}{|c|}{ Univariable } & \multicolumn{3}{|c|}{ Multivariable Model 1} & \multicolumn{3}{|c|}{ Multivariable Model 2} \\
\hline & $\beta$ & $95 \% \mathrm{Cl}$ & $p$ & $\beta$ & $95 \% \mathrm{Cl}$ & $p$ & $\beta$ & $95 \% \mathrm{Cl}$ & $p$ \\
\hline EDSS & 0.269 & $0.125,0.413$ & $<0.001$ & 0.256 & $0.108,0.404$ & 0.001 & 0.249 & $0.096,0.401$ & 0.001 \\
\hline RRMS $\vee$ PMS & 0.198 & $0.048,0.347$ & 0.010 & 0.407 & $0.158,0.655$ & 0.001 & 0.292 & $-0.062,0.647$ & 0.106 \\
\hline BPF & -0.006 & $-0.010,-0.003$ & $<0.001$ & -0.003 & $-0.006,0.000$ & 0.043 & -0.003 & $-0.006,0.000$ & 0.040 \\
\hline T2LV & 0.035 & $-0.001,0.071$ & 0.055 & 0.019 & $-0.019,0.057$ & 0.329 & 0.031 & $-0.009,0.071$ & 0.124 \\
\hline
\end{tabular}

Legend: EDSS Expanded disability status scale, RRMS Relapsing-remitting multiple sclerosis, PMS Progressive multiple sclerosis, BPF Brain parenchymal fraction, T2LV Cerebral T2-hyperintense lesion volume. All statistical models are linear mixed effects regressions. Multivariable model \#1 includes the following covariates: age, disease duration, gender, and specific DMT use. Model \#2 includes the covariates: age, disease duration, gender, smoking history, specific DMT use, CES-D score, MSSS score, SF-36 physical and mental composite scores

(model 2) using age, disease duration, gender, smoking history, DMT, depression, social support, and quality of life measures as covariates $(\beta=0.45, p=0.003)$. With BPF as an outcome, higher MLR, but not NLR, was significantly associated with greater whole brain atrophy in all statistical models $(p<0.05)$. Lastly, neither NLR or MLR were good correlates of T2 lesion volume, although univariable comparison with MLR was borderline ( $p=$ 0.055). We did not include MFIS in the multivariable model due to the decreased sample size $(N=424)$; when we did include it, results (model \#2) were similar. Given the strong associations with DMT (Table 2), we also performed sensitivity analyses using untreated patients only $(N=146)$, and found 3-7 times increase in effect size for associations with EDSS and disease category (relapsing vs. progressive MS) in all linear models: see Additional file 1 for full information.

\section{NLR/MLR does not predict longitudinal worsening of disability}

Using only subjects with longitudinal data $(N=329$, 1279 total observations), we tested whether baseline (first measurement) NLR or MLR could predict subsequent worsening of EDSS using the interaction term: (time elapsed)*(ln)M/NLR. This interaction was neither significant in all subjects (NLR $p=0.15 ; \operatorname{MLR} p=0.22$ ), nor a subset of early relapsing patients (disease duration $<5$ years, $N=144$, NLR $p=0.77$; MLR $p=0.49$ ), nor a cohort of early relapsing untreated patients $(N=27$, NLR $p=0.08 ; \operatorname{MLR} p=0.64$ ).

\section{Discussion}

The field of multiple sclerosis continues to lack a comprehensive serum biomarker for the diagnosis or monitoring of disease activity. Here we present evidence that the common hematologic index ratios of neutrophil-to-lymphocyte (NLR) and monocyte-to-lymphocyte (MLR) are both robustly associated with neurological disability scores in MS, independent of all demographic and clinical variables. Additionally, the MLR is associated with the MRI outcome of whole brain atrophy (BPF), but not inflammatory burden of T2-hyperintense cerebral lesions (T2LV). A higher NLR (and MLR to a lesser extent) associate strongly with a diagnosis of progressive disease in MS. NLR, but not MLR, is additionally associated with self-reported patient outcomes including depression, fatigue, and quality of life, and thus represents a psycho-neuro-immunological marker. Strengths of this study include the large and well-characterized sample of MS patients, including self-reported psychological outcome measures, specific DMT use, and quantified neuroimaging pathology. Together this dataset allows robust statistical modeling and higher confidence in the results; to our knowledge, this is the largest report of NLR in multiple sclerosis, the first to address confounding with DMT use, and include characterization of neuroimaging findings; this is the first reporting of the MLR in MS as well.

Our findings generally corroborate the limited prior literature in this specific area, which consists of four case-control, and one retrospective cohort study. All case-control studies found significantly elevated NLR in MS (or optic neuritis) compared to controls [13, 20, 22]. All groups found a mean NLR $>2.1$ in MS compared to healthy controls NLR $<2.1$, however we urge caution before directly comparing trials since we found the $\mathrm{CBC}$ analysis machine to be a significant source of variability in our data and therefore excluded the use of the SYSMEX XE machine (results not shown). This methodological observation limits inter-study comparisons, and carries implications for the role of NLR/MLR as potential biomarkers. Several of these prior studies also found a higher NLR during flares compared to remission. We did not attempt to replicate those results in our data due to the uncertain timing of (likely) intravenous glucocorticoid administration during flares; this medication would significantly elevate the NLR through demargination of neutrophils and we therefore excluded all $\mathrm{CBC}$ measurements during flares.

Three studies have looked at the role of NLR as a correlate of disability in MS; two of these demonstrated a strong univariable [21] or multivariable [20] association, discriminating EDSS scores of 5 and 3, respectively; whereas one group found no significant value of NLR to predict EDSS $>4$ in a cross-sectional multivariable 
model including age, gender, and disease duration [22]; this latter study also found no association with clinical phenotype (relapsing vs progressive MS). Here, our results support a strong association between higher NLR/MLR and worse disability scores in robust multivariable models; these measures are similarly able to significantly discriminate progressive from relapsing patients. However, despite robust correlations with disability and substantial prior literature suggesting a role for the NLR in prognostication, a limited longitudinal analysis did not reveal a role for NLR or MLR in predicting future disability worsening. Our retrospective analysis was not properly designed to answer this question however, so this data should be interpreted cautiously and further investigation is warranted.

The disease-modifying treatments used in the contemporary treatment of MS are, by design, highly immunologically active and clearly affect the NLR and MLR. Most DMTs except glatiramer acetate have well-established (and possibly nonlinear) effects on the numbers of circulating leukocytes; most relevant here are fingolimod (lymphopenia/monocytosis) [31], natalizumab (lymphocytosis) [32], cyclophosphamide (lymphopenia), and interferon-beta (lymphopenia/ neutropenia) [33], effects that are confirmed from our data (Table 2). Overall these medications clearly limit the utility of the NLR/MLR as cross-sectional bedside translational markers- a challenge with any biomarker in MS. However, these effects may be accounted for as covariates as we did here; sensitivity analyses using only untreated patients typically made the associations between NLR/MLR and MS outcomes such as EDSS even more robust (see Additional file 1). Ours is the only study in the literature to statistically account for the effects of DMT, and our results therefore suggest a possible role in clinical translation, especially given the low cost and widespread accessibility of this marker.

Lastly, Al-Hussain et al. [13] focused their paper on the significant associations between NLR and psychological stress $(p<0.001)$. Whereas they did not find significant associations with depression or anxiety, our analysis demonstrated a small but highly significant association between NLR and depression after controlling for age, gender, smoking history and DMT, similar to results of a recent meta-analysis of NLR in mood disorders [14]. We could not replicate prior findings of an association between MLR and social support [34]. These psychoneuroimmunological associations should be further explored as potential avenues of treatment that interact with neuroinflammation.

More work is needed to better elucidate the mechanistic immune feedback loops that presumably underlie the origin of absolute myeloid and lymphocyte counts in the circulating blood compartment; especially regarding the (temporal) dynamics involved in relative rates of leukocyte production, sequestration, egress, and survival dynamics which are all integrated into these markers. In a general sense, the numerator represents nonspecific activation of the innate (myeloid) immune system, and the denominator reflects suppression of more adaptive processes. Higher circulating monocytes (MLR) were associated with greater whole brain atrophy and also with male gender, the latter being a well-known risk for more rapid disability accumulation [35]. The observation that the NLR and MLR are significantly associated with neuroinflammation-related disability, disease category, and brain atrophy outcomes, also reflects current thinking in the multiple sclerosis literature regarding a transition from adaptive to (upregulated) innate immune processes during the conversion phase from relapsing to progressive clinical disease [36, 37]. This clinical transition is also marked by a plateau effect of T2-hyperintense lesions [38], a finding likely reflected in our data by the lack of association between NLR/MLR and T2LV.

Multiple limitations exist in this study, perhaps most importantly that our results are of an associative nature only, and thus no conclusions should be drawn regarding causality. Complex bidirectional relationships exist regarding the connection between psychosocial (patient-reported) factors, immune function, and disease outcomes; our study only provides a limited insight into how these factors interact. Additionally this study is limited by its retrospective nature that is susceptible to selection and information biases; these include unclear motivations for ordering CBCs among untreated patients, or those on glatiramer acetate in whom hematological monitoring is not required. Patients in the untreated group may still be affected by prior drug washout. Some of our cohort is missing neuroimaging outcome data or patient-reported outcomes, which reduces power in certain analyses and may bias results. Further, we lack insight into subjects' medical comorbidities or other medication use which may affect the NLR/MLR. Lastly, the CBC-d only provides a gross metric of immune function and is limited in resolving lympocyte subsets such as Th1/ Th17 that are important in MS pathogenesis $[39,40]$.

\section{Conclusions}

As a readily available and widespread diagnostic and monitoring tool, the NLR and MLR may represent translational metrics that can provide independent and complementary cross-sectional information to a patient's inflammatory disease status in multiple sclerosis.

\section{Additional file}

\footnotetext{
Additional file 1: Tables S1-S4 Sensitivity analyses using cross-sectiona data only, using a single time point (subjects' last clinic visit), and are otherwise analogous to Tables 2, 3, 4 and 5 in the manuscript. Tables S5. and S6. Sensitivity analyses using a subset of patients who were untreated (not on a disease-modifying therapy) at the time of their clinical visit. (DOCX 53 kb)
} 


\section{Abbreviations}

BMl: Body mass index; BPF: Brain parenchymal fraction; CBC: Complete blood count; CBC-d: Complete blood count with differential; CES-D: Center for Epidemiology Depression scale; CLIMB: Comprehensive Longitudinal Investigation of Multiple sclerosis in Boston; CNS: Central nervous system; DMT: Disease-modifying therapy; EDSS: Expanded disability status scale; MFIS: Modified Fatigue Inventory Scale; MLR: Monocyte-to-lymphocyte ratio; MRI: Magnetic resonance imaging; MS: Multiple sclerosis; MSSS: Modified Study Social Support Survey; NLR: Neutrophil-to-lymphocyte ratio; PRO: Patient-reported outcome; T2LV: Cerebral T2-hyperintense lesion volumes

\section{Acknowledgements}

We thank Svetlana Egorova, Cindy Gonzalez, and Mariann Polgar-Turcsanyi for technical assistance.

\section{Funding}

This work was supported by a Clinical Research Training Fellowship in Multiple Sclerosis to Dr. Hemond from the American Academy of Neurology Institute with funding provided in part by Sanofi-Genzyme. Funding agencies had no involvement in this work

\section{Availability of data and materials}

The datasets generated and/or analyzed during the current study are not publicly available due to reasons of patient confidentiality and CLIMB proprietary information; this dataset may be available from the corresponding author on reasonable request.

\section{Authors' contributions}

$\mathrm{CH}$ : Conceived and designed the study, acquired and analyzed data, wrote manuscript; BG: Data analysis and interpretation, involved in drafting and revision of the manuscript; RB: Revised manuscript for critically important intellectual content and data analysis; TC: Data acquisition and interpretation, revised the manuscript for critically important intellectual content; $\mathrm{BH}$ : Statistical data analysis and interpretation, manuscript drafting and revision, substantial contributions to study design. All authors have read and approved this manuscript.

\section{Ethics approval and consent to participate}

This study was reviewed and approved by the Partners Human Research Committee ethics board at the Brigham and Women's Hospital (IRB Protocol \# 1999-P-010435). All research subjects provided informed written consent.

\section{Consent for publication}

Not applicable.

\section{Competing interests}

The authors declare that they have no competing interests.

\section{Publisher's Note}

Springer Nature remains neutral with regard to jurisdictional claims in published maps and institutional affiliations.

\section{Author details}

'Department of Neurology, University of Massachusetts Medical Center, 55 Lake Ave North, Worcester, MA 01655, USA. ²Department of Neurology, Harvard Medical School, Boston, MA, USA. ${ }^{3}$ Partners Multiple Sclerosis Center, Ann Romney Center for Neurologic Diseases, Brigham \& Women's Hospital, Boston, MA, USA. ${ }^{4}$ Department of Radiology, Harvard Medical School, Boston, MA, USA.

Received: 2 August 2018 Accepted: 30 January 2019

Published online: 12 February 2019

\section{References}

1. Hemmer B, Kerschensteiner M, Korn T. Role of the innate and adaptive immune responses in the course of multiple sclerosis. Lancet Neurol. 2015; 14:406-19. https://doi.org/10.1016/S1474-4422(14)70305-9.

2. Rumble JM, Huber AK, Krishnamoorthy G, Srinivasan A, Giles DA, Zhang X, et al. Neutrophil-related factors as biomarkers in EAE and MS. J Exp Med. 2015;212:23-35. https://doi.org/10.1084/jem.20141015.
3. Ocana A, Nieto-Jiménez C, Pandiella A, Templeton AJ. Neutrophils in cancer: prognostic role and therapeutic strategies. Mol Cancer. 2017;16:1-7. https:// doi.org/10.1186/s12943-017-0707-7.

4. Nishijima TF, Muss HB, Shachar SS, Tamura K, Takamatsu Y. Prognostic value of lymphocyte-to-monocyte ratio in patients with solid tumors: a systematic review and meta-analysis. Cancer Treat Rev. 2015;41:971-8. https://doi.org/ 10.1016/j.ctrv.2015.10.003.

5. Afari ME, Bhat T. Neutrophil to lymphocyte ratio (NLR) and cardiovascular diseases: an update. Expert Rev Cardiovasc Ther. 2016;14:573-7.

6. Fu H, Qin B, Hu Z, Ma N, Yang M, Wei T, et al. Neutrophil- and platelet-tolymphocyte ratios are correlated with disease activity in rheumatoid arthritis. Clin Lab. 2015;61:269-73.

7. Tokgoz S, Kayrak M, Akpinar Z, Seyithanoğlu A, Güney F, Yürüten B. Neutrophil lymphocyte ratio as a predictor of stroke. J Stroke Cerebrovasc Dis. 2013;22:1169-74.

8. Lattanzi S, Cagnetti C, Provinciali L, Silvestrini M. Neutrophil-to-lymphocyte ratio predicts the outcome of acute intracerebral hemorrhage. Stroke. 2016; 47:1654-7. https://doi.org/10.1161/STROKEAHA.116.013627.

9. Kuyumcu ME, Yesil Y, Oztürk ZA, Kizilarslanoǧlu C, Etgül S, Halil M, et al. The evaluation of neutrophil-lymphocyte ratio in Alzheimer's disease. Dement Geriatr Cogn Disord. 2012;34:69-74.

10. Kalelioglu T, Yuruyen M, Gultekin G, Yavuzer H, Özturk Y, Kurt M, et al. Neutrophil and platelet to lymphocyte ratios in people with subjective, mild cognitive impairment and early Alzheimer's disease. Psychogeriatrics. 2017; 17:506-8.

11. Akıl E, Bulut A, Kaplan I, Özdemir HH, Arslan D, Aluçlu MU. The increase of carcinoembryonic antigen (CEA), high-sensitivity C-reactive protein, and neutrophil/lymphocyte ratio in Parkinson's disease. Neurol Sci. 2015;36:423-8.

12. Dhabhar FS, Miller AH, McEwen BS, Spencer RL. Stress-induced changes in blood leukocyte distribution. Role of adrenal steroid hormones. J Immunol. 1996;157:1638-44

13. Al-Hussain F, Alfallaj MM, Alahmari AN, Almazyad AN, Alsaeed TK, Abdurrahman AA, et al. Relationship between neutrophil-to-lymphocyte ratio and stress in multiple sclerosis patients. J Clin Diagn Res. 2017:11: CC01-4. https://doi.org/10.7860/JCDR/2017/24388.9764.

14. Mazza MG, Lucchi S, Tringali AGM, Rossetti A, Botti ER, Clerici M. Neutrophil/ lymphocyte ratio and platelet/lymphocyte ratio in mood disorders: a metaanalysis. Prog Neuro-Psychopharmacol Biol Psychiatry. 2018;84:229-36.

15. Serfőző G, Horváth T, Földesi I, Rafael B, von Känel R, Keresztes M. The monocyte-to-lymphocyte ratio correlates with psycho-neuro-inflammatory factors in patients with stable coronary artery disease. Neuroimmunomodulation. 2016;23:67-74. https://doi.org/10.1159/000443835.

16. Kern S, Ziemssen T. Brain-immune communication psychoneuroimmunology of multiple sclerosis. Mult Scler. 2008;14:6-21.

17. Frank MG, Weber MD, Watkins LR, Maier SF. Stress-induced neuroinflammatory priming: a liability factor in the etiology of psychiatric disorders. Neurobiol Stress. 2016;4:62-70. https://doi.org/10.1016/j.ynstr.2015. 12.004

18. Wohleb ES, Franklin T, Iwata M, Duman RS. Integrating neuroimmune systems in the neurobiology of depression. Nat Rev Neurosci. 2016;17:497511. https://doi.org/10.1038/nrn.2016.69.

19. Naegele M, Tillack K, Reinhardt S, Schippling S, Martin R, Sospedra M. Neutrophils in multiple sclerosis are characterized by a primed phenotype. J Neuroimmunol. 2012;242:60-71. https://doi.org/10.1016/j.jneuroim.2011.11.009.

20. Demirci SS, Demirci SS, Kutluhan S, Koyuncuoglu HR, Yurekli VA. The clinical significance of the neutrophil-to-lymphocyte ratio in multiple sclerosis. Int J Neurosci. 2016;126:700-6. https://doi.org/10.3109/00207454.2015.1050492.

21. Guzel I, Mungan S, Oztekin ZN, Ak F. Is there an association between the expanded disability status scale and inflammatory markers in multiple sclerosis? J Chinese Med Assoc. 2016;79:54-7.

22. Bisgaard AK, Pihl-jensen G, Frederiksen JL. The neutrophil-to-lymphocyte ratio as disease actvity marker in multiple sclerosis and optic neuritis. Mult Scler Relat Disord. 2017;18:213-7. https://doi.org/10.1016/j.msard.2017.10.009.

23. Hemond C, Glanz B, Bakshi R, Chitnis T, Healy B. The neutrophil-tolymphocyte and monocyte-to-lymphocyte ratios are independently associated with neurological disability and brain atrophy in multiple sclerosis. Poster Presentation at: Eur Comm Treat Res Mult Sclerosis, Berlin, Germany. 2018;P876. https://doi.org/10.1177/1352458518798590.

24. Gauthier SA, Glanz BI, Mandel M, Weiner HL. A model for the comprehensive investigation of a chronic autoimmune disease: the multiple sclerosis CLIMB study. Autoimmun Rev. 2006;5:532-6. 
25. Polman CH, Reingold SC, Edan G, Filippi M, Hartung H-P, Kappos L, et al. Diagnostic criteria for multiple sclerosis: 2005 revisions to the "McDonald Criteria". Ann Neurol. 2005;58:840-6.

26. Radloff LS. The CES-D scale: a self-report depression scale for research in the general population. Appl Psychol Meas. 1977;1:385-401. https://doi.org/10. 1177/014662167700100306

27. Larson RD. Psychometric properties of the modified fatigue impact scale. Int J MS Care. 2013;15:15-20.

28. Ware JJ, Sherbourne C. The MOS 36-item short-form health survey (SF-36). I. Conceptual framework and item selection. Med Care. 1992;30:473-83.

29. Sherbourne CD, Stewart AL. The MOS social support survey. Soc Sci Med. 1991;32:705-14.

30. Wei X, Warfield SK, Zou KH, Wu Y, Li X, Guimond A, et al. Quantitative analysis of MRI signal abnormalities of brain white matter with high reproducibility and accuracy. J Magn Reson Imaging. 2002:15:203-9.

31. Nakhaei-Nejad M, Barilla D, Lee C-H, Blevins G, Giuliani F. Characterization of lymphopenia in patients with MS treated with dimethyl fumarate and fingolimod. Neurol Neuroimmunol Neuroinflamm. 2018;5:e432. https://doi. org/10.1212/NXI.0000000000000432.

32. Bridel C, Beauverd Y, Samii K, Lalive PH. Hematologic modifications in natalizumab-treated multiple sclerosis patients: an 18-month longitudinal study. Neurol Neuroimmunol Neuroinflamm. 2015;2:e123. https://doi.org/10. 1212/NXI.0000000000000123.

33. Rieckmann P, O'Connor P, Francis GS, Wetherill G, Alteri E. Haematological effects of interferon-beta-1a (Rebif) therapy in multiple sclerosis. Drug Saf. 2004;27:745-56.

34. Gidron Y, Armon T, Gilutz H, Huleihel M. Psychological factors correlate meaningfully with percent-monocytes among acute coronary syndrome patients. Brain Behav Immun. 2003;17:310-5.

35. Ribbons KA, McElduff P, Boz C, Trojano M, Izquierdo G, Duquette P, et al. Male sex is independently associated with faster disability accumulation in relapseonset MS but not in primary progressive MS. PLoS One. 2015;10:1-11.

36. Weiner $\mathrm{HL}$. The challenge of multiple sclerosis: how do we cure a chronic heterogeneous disease? Ann Neurol. 2009;65:239-48.

37. Mishra MK, Yong WW. Myeloid cells — targets of medication in multiple sclerosis. Nat Rev Neurol. 2016;12:539-51. https://doi.org/10.1038/nrneurol. 2016.110.

38. Li DKB, Held U, Petkau J, Daumer M, Barkhof F, Fazekas F, et al. MRI T2 lesion burden in multiple sclerosis: a plateauing relationship with clinical disability. Neurology. 2006;66:1384-9.

39. Rostami A, Ciric B. Role of Th17 cells in the pathogenesis of CNS inflammatory demyelination. J Neurol Sci. 2013;333:76-87. https://doi.org/10 1016/j.jns.2013.03.002.

40. Van Langelaar J, Van Der Vuurst De Vries RM, Janssen M, Wierenga-Wolf AF, Spilt IM, Siepman TA, et al. Thelper 17.1 cells associate with multiple sclerosis disease activity: perspectives for early intervention. Brain. 2018;141: 1334-49.

Ready to submit your research? Choose BMC and benefit from:

- fast, convenient online submission

- thorough peer review by experienced researchers in your field

- rapid publication on acceptance

- support for research data, including large and complex data types

- gold Open Access which fosters wider collaboration and increased citations

- maximum visibility for your research: over $100 \mathrm{M}$ website views per year

At $\mathrm{BMC}$, research is always in progress.

Learn more biomedcentral.com/submissions 